\title{
Combined treatment with sitagliptin and vitamin $D$ in a patient with latent autoimmune diabetes in adults
}

\author{
E Rapti', S Karras', M Grammatiki', A Mousiolis', X Tsekmekidou', E Potolidis', \\ P Zebekakis', M Daniilidis² and K Kotsa'
}

'Diabetes Center of 1st Department of Internal Medicine, AHEPA University Hospital, Thessaloniki, Greece and 21st Department of Internal Medicine, AHEPA University Hospital, Thessaloniki, Greece

Correspondence should be addressed to K Kotsa

Email

kalmanthou@yahoo.gr

\section{Summary}

Latent autoimmune diabetes in adults (LADA) is a relatively new type of diabetes with a clinical phenotype of type 2 diabetes (T2D) and an immunological milieu characterized by high titers of islet autoantibodies, resembling the immunological profile of type 1 diabetes (T1D). Herein, we report a case of a young male, diagnosed with LADA based on both clinical presentation and positive anti-glutamic acid decarboxylase antibodies (GAD-abs), which were normalized after combined treatment with a dipeptidyl peptidase-4 inhibitor (DPP-4) (sitagliptin) and cholecalciferol.

\section{Learning points:}

- Anti-glutamic acid decarboxylase antibodies (GAD-abs) titers in young patients being previously diagnosed as type 2 diabetes (T2D) may help establish the diagnosis of latent autoimmune diabetes in adults (LADA).

- Sitagliptin administration in patients with LADA might prolong the insulin-free period.

- Vitamin D administration in patients with LADA might have a protective effect on the progression of the disease.

\section{Background}

Latent autoimmune diabetes in adults (LADA) is a slowly progressive form of autoimmune diabetes mellitus characterized by older age at diagnosis compared with type 1 diabetes (T1D) and the presence of pancreatic islet cell autoantibodies (1).

This results in the development of glucose intolerance and overt clinical disease when the majority of pancreatic cells are not functional due to the chronic autoimmune inflammation. This pathophysiological process is characterized by the presence of circulating antibodies against pancreatic islet cells and islet cell infiltration by mononuclear lymphocytes. Commonly, patients with LADA present with a relatively preserved beta-cell function compared with patients with T1D, they manifest a progressive deterioration of beta-cell function necessitating basal and prandial insulin therapy early after diagnosis (1).

Although, LADA is genetically associated with T1D, it shares some clinical features with type 2 diabetes (T2D). Usually, LADA patients are older than 30 years, have higher BMI in comparison with T1D patients, and because initially they maintain residual insulin production are often misdiagnosed as T2D. However, in clinical practice, LADA patients are younger at diagnosis than T2D, they tend to have worse glycemic control, lower BMI, and frequently insulin treatment is initiated much earlier than 
T2D (1). Hence, in rare instances, LADA patients present solely with diabetic ketoacidosis (2; Table 1$)$.

In this report, we describe the case of a young male diagnosed with LADA based on both clinical presentation and positive anti-glutamic acid decarboxylase antibodies (GAD-abs), which were normalized after combined treatment with a dipeptidyl peptidase-4 inhibitor (DPP-4) inhibitor (sitagliptin) and cholecalciferol.

\section{Case presentation}

A 31-year-old Caucasian male was referred to the emergency department with symptoms of vomiting and nausea. He had a 3 month history of polyuria and polydipsia, accompanied by a $15 \mathrm{~kg}$ weight loss and blurred vision.

He reported no remarkable previous medical conditions, except being a heavy smoker since adolescence. He was obese with a BMI of $32.8 \mathrm{~kg} / \mathrm{m}^{2}$. His family history revealed the presence of autoimmune disorders, since his sister had been diagnosed with T1D at the age of 5 , autoimmune hemolytic anemia and autoimmune thyroiditis during puberty and had undergone thymoma resection at the age of 22 .

\section{Investigation}

On initial physical examination, the patient's arterial blood pressure was systolic: $105 \mathrm{mmHg}$ /diastolic: $65 \mathrm{mmHg}$, with a pulse rate of 104 b.p.m and a respiratory rate of 24 breaths per minute. Axillary temperature was $37.1^{\circ} \mathrm{C}$, but no obvious clinical signs of infection were found on the physical examination. Arterial $\mathrm{pH}$ was within normal range (7.36), urine analysis was negative for leukocytes, white blood cells (WBC) count was normal, and no radiologic signs of infection were found. Laboratory investigation demonstrated the following pathologic findings: blood glucose level of $300 \mathrm{mg} / \mathrm{dL}$ with traces of ketones in urine and glycosylated hemoglobin (HbA1c) at 9.6\%.
The patient declined insulin therapy and was initially treated with gliclazide once daily and metformin $1000 \mathrm{mg}$ twice daily with the suggestion of regular consultation in an outpatient diabetes clinic.

On presentation to the diabetes outpatient setting 3 weeks later, he had poor glycemic control, with fasting blood glucose values of approximately $180 \mathrm{mg} / \mathrm{dL}$ and a BMI of $28.6 \mathrm{~kg} / \mathrm{m}^{2}$. As LADA was suspected due mainly to the family history, GAD-abs titer was measured since islet autoantibodies and IA-2 antibodies are rarely detected in LADA (3). Results demonstrated a positive titer at $32 \mathrm{U} / \mathrm{mL}$ (six times the upper limit) (GAD-abs normal values $<5 \mathrm{U} / \mathrm{mL}$ ) (Table 2 ). HLA genotyping for $D R$ - and $D Q$-encoding loci were performed and results were HLA-DRB1 *04:01/03:01 and HLA-DQB1 *02:01/03:02. By combining all available clinical and laboratory data including patient's age, increased GAD-abs titer, and the haplotype of DQB1*02:01/03:02, which has been reported to be positively associated with T1D, the diagnosis of LADA was supported (4). Further biochemical workup revealed normal findings with the exception of profound vitamin D hypovitaminosis (25-OH-D): $11 \mathrm{ng} / \mathrm{mL}$ (vitamin D normal values $>30$ ) (Table 2 ). The patient was referred to an ophthalmologist for funduscopy, which revealed no signs of diabetic retinopathy.

\section{Treatment}

As the patient declined insulin therapy again, he was advised to discontinue gliclazide treatment and a combination of metformin $850 \mathrm{mg} /$ sitagliptin $50 \mathrm{mg}$ twice daily along with vitamin D supplementation (cholecalciferol p.o., $2000 \mathrm{IU} /$ daily) was prescribed.

\section{Outcome and follow-up}

Upon completion of the first month of treatment, the patient started to show a significant decrease in his blood glucose values, until complete glycemic control 8 weeks

Table 1 Clinical presentation of T1D, T2D, and LADA.

\begin{tabular}{|c|c|}
\hline Clinical characteristics & T1D \\
\hline Autoantibodies & Positive \\
\hline Pathophysiology & $\begin{array}{l}\text { Autoimmune destruction of } \\
\text { pancreatic beta-cell }\end{array}$ \\
\hline Insulin requirements & From the time of diagnosis \\
\hline Ketoacidosis & Usually with diagnosis \\
\hline
\end{tabular}

\section{LADA}

Positive

Autoimmune destruction of

pancreatic beta-cell

Soon after diagnosis

When patient is insulinopenic
T2D

Negative

Increased insulin resistance, reduced insulin production

Late after diagnosis, when oral antidiabetic agents no longer maintain adequate glycemic control Usually absent, aggravated by other comorbidities 
Table 2 Laboratory investigation from initial diagnosis to 2 years later.

\begin{tabular}{|c|c|c|c|c|}
\hline & Day of diagnosis & Diabetes clinic visit & One year after diagnosis & Two years after diagnosis \\
\hline $\mathrm{HbA1c}$ & $9.6 \%$ & & $5.4 \%$ & $5.2 \%$ \\
\hline GAD-abs (U/mL) & & 32 & 4.2 & 4.1 \\
\hline Creatinine (mg/dL) & 1.1 & & 1.2 & 1.1 \\
\hline Potassium (mEq/L) & 4.9 & 4.1 & & 4.5 \\
\hline Sodium (mEq/L) & 142 & 140 & & 139 \\
\hline SGOT (U/L) & 19 & & 16 & 17 \\
\hline SGPT (U/L) & 27 & & 20 & 19 \\
\hline Cholesterol (mg/dL) & & 241 & 194 & 216 \\
\hline Triglycerides (mg/dL) & & 157 & 85 & 99 \\
\hline $\mathrm{TSH}(\mu \mathrm{IU} / \mathrm{mL})$ & & 1.01 & 1.3 & 1.21 \\
\hline Insulin $(\mu \mathrm{IU} / \mathrm{mL})$ & & 10.3 & & \\
\hline C-peptide $(\mathrm{mg} / \mathrm{mL})$ & & 1.0 & & \\
\hline Cortisol ( $\mu \mathrm{g} / \mathrm{dL})$ & & 16.9 & & \\
\hline Urine & Ketone traces & & & \\
\hline
\end{tabular}

SGOT, serum glutamic oxaloacetic transaminase; SGPT, serum glutamic pyruvic transaminase; TSH, thyroid-stimulating hormone.

after diagnosis, reaching fasting plasma glucose concentrations between 70 and $120 \mathrm{mg} / \mathrm{dL}$ and an HbA1c of $7.3 \%$. Being able to comply with diet and exercise consultation, he presented 6 months later with an excellent glycemic profile and an HbA1c of $6.1 \%$. By this time, the patient had lost another $7 \mathrm{~kg}$ of weight and his BMI was $24.9 \mathrm{~kg} / \mathrm{m}^{2}$.

One year after diagnosis he underwent a clinical and laboratory examination, which revealed a decline to his GAD-abs levels by $86 \%$ falling within the normal range (4.2 U/mL) and his HbA1c was 5.4\% (Table 2). Currently, 2 years from initial diagnosis, under the same treatment he has negative GAD-abs, his HbA1c is $5.2 \%$, and he maintains an excellent glycemic profile.

\section{Discussion}

LADA is manifested with the same genetic and immunological profile as T1D, but it also shares common clinical features with T2D. Although our patient presented with hyperglycemia and traces of ketones in the urine, his arterial $\mathrm{pH}$ excluded the diagnosis of ketoacidosis and made the LADA diagnosis more probable. In the literature, there have occasionally been described cases of LADA patients presenting with severe insulinopenia and diabetic ketoacidosis (2), which was not the case in our patient. On the other hand, some T1D patients after initiation of insulin treatment and despite disease progression transiently regain insulin production from pancreatic cell. During, this period, which is frequently referred as a 'honeymoon period', patients present with improved glycemic control and their requirements in medication are diminishing. The incidence of remission in T1D almost always follows initial insulin treatment, is highly variable regarding the degree and the duration, and is more common in the adult population within the first year of diagnosis (5). In this case although insulin was never administered, a notable and fast improvement was evident soon after treatment initiation and was maintained for 2 years thereafter.

The pathogenesis of LADA involves the presence of autoantibodies and the progressive deterioration of the beta-cell function (6). Diagnosis is primarily based on three criteria: (i) age of disease onset $\geq 30$ years, (ii) detection of high titers in at least one of anti-islet antibodies and (iii) no need of insulin treatment early after diagnosis $(4,7)$.

Our patient was diagnosed with LADA based on his age, positive GAD-abs, and HLA alleles that are linked to T1D. Although $10 \%$ of patients with presumed T2D at diagnosis, in fact, have positive antibodies, therapeutic guidelines regarding this have not yet been established $(4,7)$. An ideal therapeutic approach would aim not only at diminishing the acute and chronic complications associated with diabetes, but also at preserving beta-cell residual function.

Sitagliptin is a potent DPP-4 inhibitor which results in the delay of degradation of incretin hormones (glucagon-like peptide-1 (GLP-1) and gastric inhibitory peptide (GIP)), thereby improving beta-cell function and resulting in better glycemic control in patients with T2D. Nevertheless, this DPP-4 inhibitor was prescribed to our patient due to a growing body of evidence that attributes immunomodulatory effects to this pharmaceutical compound. Recent studies demonstrate that the use of sitagliptin in individuals with T1D improved overall the glycemic control and reduced insulin requirements 
without altering the amount of endogenous insulin production (8). Although great controversy exists in available data $(8,9)$, it has been proven that DPP-4 (also known as adenosine deaminase complexing protein 2 or CD26) is a transmembrane glycoprotein encoded by the DPP4 gene. CD26 is expressed by many cell types including lymphocytes and is associated with immune regulation. Sitagliptin by inhibiting DPP-4/CD26 decreased the incidence of T1D in the non-obese diabetic (NOD) mouse by reducing CD4 T-cell migration (10). Recently, case reports have described DPP-4 inhibition effects on patients speculating an immunomodulatory pathway. A patient diagnosed with T1D attained an excellent glycemic control without insulin, only by administration of $100 \mathrm{mg}$ of sitagliptin daily 15 months after initial diagnosis (11). In another recent report in a T2D patient with a 17-year history of psoriasis, a gradual remission of his skin lesions was reported after sitagliptin treatment initiation (12). A prospective study of 1-year duration demonstrated maintenance of beta-cell function through the administration of sitagliptin in patients with recent-onset LADA (13). A similar study proved that c-peptide secretion and glycemic control was improved by the use of another DPP-4 inhibitor, saxagliptin, versus placebo in LADA patients (14). All these accumulating evidences support the hypothesis that this may be a class effect; however, further studies are required.

In our case, the patient received additional oral supplementation with vitamin $\mathrm{D}$, due to a deficiency that was revealed in laboratory investigation. The active form of vitamin $\mathrm{D},\left[1.25(\mathrm{OH})_{2} \mathrm{D}_{3}\right]$, regulates not only calcium metabolism, but also modulates the activity of different defense and immune cells, including lymphocytes, monocytes, macrophages and epithelial cells. Since vitamin D3 promotes phagocytosis by triggering macrophages and affects immune response, it is potentially involved in the pathogenesis of several diseases. A positive correlation between T1D mellitus prevalence and hypovitaminosis $\mathrm{D}$ has been demonstrated in several epidemiological trials (15). Taking into consideration these findings, researchers tested the hypothesis that similar results will emerge from the use of vitamin D in LADA patients. Positive correlation was found between 1-alpha $(\mathrm{OH}) \mathrm{D} 3$ plus insulin and the preservation of betacell function in early onset LADA (16). Moreover, findings from animal trials suggested that low levels of vitamin D may cause a reduction in the secretion of insulin by the beta-cell. Supplementation with $1.25(\mathrm{OH})_{2} \mathrm{D}_{3}$ in patients with T1D with hypovitaminosis $\mathrm{D}$ has been shown to improve their glycemic profile $(17,18)$. In our case, the patient experienced a remarkable decline in GAD-abs titer, which contributed to his excellent glycemic profile for two consecutive years. In the literature, only few data exist to correlate the use of vitamin D with the reduction of GAD-abs in children with T1D. Further investigation in larger cohorts has been advocated to draw safer conclusions (19).

Both vitamin D analogs and DPP-4 inhibitors have been shown to improve beta-cell function and attenuate autoimmunity in diabetes. To our knowledge, this is the first case of combined treatment in a patient with LADA that resulted in reverting the phenotype and preserving excellent glycemic control without the use of insulin 24 months after initial diagnosis.

Declaration of interest

The authors declare that there is no conflict of interest that could be perceived as prejudicing the impartiality of the research reported.

\section{Funding}

This research did not receive any specific grant from any funding agency in the public, commercial or not-for-profit sector.

\section{Patient consent}

Written informed consent was obtained from the patient for publication of this case report.

\section{Author contribution statement}

E Rapti is a trainee doctor in Endocrinology, who treated the patient and prepared the initial manuscript. K Kotsa is the Assistant Professor of the department, who supervised the care of the patient and edited the manuscript. All other authors have provided significant contribution toward patient care.

\section{References}

1 Laugesen E, Østergaard JA \& Leslie RD 2015 Latent autoimmune diabetes of the adult: current knowledge and uncertainty. Diabetic Medicine 32 843-852. (doi:10.1111/dme.2015.32.issue-7)

2 Nadhem O, Nakhla E \& Smalligan RD 2015 Diabetic ketoacidosis as first presentation of latent autoimmune diabetes in adult. Case Reports in Medicine 2015 821397. (doi:10.1155/2015/821397)

3 Gale EA 2005 Latent autoimmune diabetes in adults: a guide for the perplexed. Diabetologia 48 2195-2199. (doi:10.1007/s00125-005-1954-5)

4 Desai M, Zeggini E, Horton V, Owen K, Hattersley A, Levy J, Walker M \& Gillespie K 2007 An association analysis of the HLA gene region in latent autoimmune diabetes in adults. Diabetologia 50 68-73. (doi:10.1007/s00125-006-0513-z)

5 Moole H, Moolie V, Mamidilalli A, Dharmapuri S, Boddireddy R, Taneja D, Sfeir H \& Gajula S 2015 Spontaneous complete remission of type 1 diabetes mellitus in an adult - review and case report. Journal of Community Hospital Internal Medicine Perspectives 528709. (doi:10.3402/jchimp.v5.28709) 
Endocrinology

Diabetes \& Metabolism

CASE REPORTS
E Rapti and others

LADA treatment with

sitagliptin and Vitamin D
ID: 15-0136; May 2016

DOI: $10.1530 / E D M-15-0136$
6 Pozzilli P \& Mario U 2001 Autoimmune diabetes not requiring insulin at diagnosis (latent autoimmune diabetes of the adult). Diabetes Care 24 1460-1467. (doi:10.2337/diacare.24.8.1460)

7 Cernea S, Buzetti R \& Pozzilli P 2009 -cell protection and therapy for latent autoimmune diabetes in adults. Diabetes Care 32 246-252. (doi:10.2337/dc09-S317)

8 Hari Kumar K, Shaikh A \& Prusty P 2013 Addition of exenatide or sitagliptin to insulin in new onset type 1 diabetes: a randomized, open label study. Diabetes Research and Clinical Practice 100 55-58. (doi:10.1016/j.diabres.2013.01.020)

9 Price J, Linder G, Li W, Zimmerman B, Rother K, Malek R, Alattar M \& Tarbell K 2013 Effects of short-term sitagliptin treatment on immune parameters in healthy individuals, a randomized placebo-controlled study. Clinical \& Experimental Immunology 174 120-128. (doi:10.1111/ cei.12144)

10 Kim J, Nian C \& Mcintosh H 2010 Sitagliptin (MK0431) inhibition of dipeptidyl peptidase IV decreases nonobese diabetic mouse CD4+ T-cell migration through incretin-dependent and -independent pathways. Diabetes 59 1739-1750. (doi:10.2337/ db09-1618)

11 Lima-Martinez M, Guerra-Alcala E, Contreras M, Nastasi J, Noble J \& Polychronakos C 2014 One year remission of type 1 diabetes mellitus in a patient treated with sitagliptin. Endocrinology, Diabetes \& Metabolism Case Reports 2014 140072. (doi:10.1530/EDM-14-0072)

12 Nishioka T, Shinohara M, Tanimoto N, Kumagai C \& Hashimoto K 2012 Sitagliptin, a dipeptidyl peptidase-IV inhibitor, improves psoriasis. Dermatology 224 20-21. (doi:10.1159/000333358)

13 Zhao Y, Yang L, Xiang Y, Liu L, Huang G, Long Z, Li X, Leslie RD, Wang X \& Zhou Z 2014 Dipeptidyl peptidase 4 inhibitor sitagliptin maintains $\beta$-cell function in patients with recent-onset latent autoimmune diabetes in adults: one year prospective study. Journal of Clinical Endocrinology \& Metabolism 99 E876-E880. (doi:10.1210/ jc.2013-3633)

14 Buzzetti R, Pozzilli P, Frederich R, Iqbal N \& Hirshberg B 2016 Saxagliptin improves glycaemic control and C-peptide secretion in latent autoimmune diabetes in adults (LADA). Diabetes/Metabolism Research and Reviews 32 289-296. (doi:10.1002/dmrr.v32.3)

15 Riachy R, Vandewalle B, Moerman E, Belaich S, Lukowiak B, Gmyr V, Muharram G, Kerr Conte J \& Pattou F 2006 1,25-Dihydroxyvitamin D3 protects human pancreatic islets against cytokine-induced apoptosis via down-regulation of the Fas receptor. Apoptosis $\mathbf{1 1}$ 151-159. (doi:10.1007/s10495-006-3558-z)

16 Li X, Liao L, Yan X, Huang G, Lin J, Lei M, Wang X \& Zhou Z 2009 Protective effects of 1-alpha-hydroxyvitamin D3 on residual beta-cell function in patients with adult-onset latent autoimmune diabetes (LADA). Diabetes/Metabolism Research and Reviews 25 411-416. (doi:10.1002/dmrr.v25:5)

17 Norman AW, Frankel JB, Heldt AM \& Grodsky GM 1980 Vitamin D deficiency inhibits pancreatic secretion of insulin. Science 209 823-825. (doi:10.1126/science.6250216)

18 Chiu KC, Chu A, Go VL \& Saad MF 2004 Hypovitaminosis D is associated with insulin resistance and beta cell dysfunction. American Journal of Clinical Nutrition 79 820-825.

19 Papadimitriou DT, Marakaki C, Fretzayas A, Nicolaidou P \& Papadimitriou A 2013 Negativation of type 1 diabetes-associated autoantibodies to glutamic acid decarboxylase and insulin in children treated with oral calcitriol. Journal of Diabetes 5 344-348. (doi:10.1111/jdb.2013.5.issue-3)

Received in final form 24 April 2016

Accepted 6 May 2016 